\title{
Adesão ao tratamento de Diabetes Mellitus tipo 1 atendidos em um pro- grama especializado em Porto Alegre*
}

\section{Adherence to treatment in diabetes mellitus type 1 treated in a specialized program in Porto Alegre}

\author{
Onilda Rubin ${ }^{1}$, Karina Azzolin², Suzana Muller ${ }^{3}$
}

\begin{abstract}
RESUMO
O Diabetes Mellitus é considerado um dos mais importantes males endócrino-metabólicos na infância, apresentando limitação e risco de vida ao portador por suas complicações cardiovasculares a longo prazo. Este trabalho refere-se a um estudo quantitativo que teve por objetivo avaliar a adesão ao tratamento por crianças em idade escolar portadoras de Diabetes Mellitus tipo 1 atendidas em um ambulatório especializado de Porto Alegre no Instituto da Criança com Diabetes (ICD). Os dados foram coletados por meio de um questionário semi-estruturado e aplicado a 25 crianças com média de 9 anos de idade. O tempo de acompanhamento pelo ambulatório foi de 1,96 $\pm 0,8$ anos e o tempo médio da doença tratada foi de $1,76 \pm 0,7$ anos. A maioria das crianças (84\%) possui os pais como principais cuidadores, todas reconhecem a importância da consulta de enfermagem no tratamento e $92 \%$ confiam nas condutas dos profissionais. Sessenta por cento das crianças enfrentam dificuldades relacionadas ao plano alimentar. A adesão ao tratamento foi observada em 11 crianças (44\%) que, além de não faltarem às consultas, também não esqueceram de aplicar insulina nos últimos 6 meses e referiram seguir a dieta prescrita. Com isso, concluímos que a aderência de crianças diabéticas ao tratamento está relacionada ao interesse dos pais pelo tratamento, ao conhecimento da doença e também aos fatores sócio-econômicos. Por fim, o trabalho da enfermeira foi plenamente reconhecido pelos pais.
\end{abstract}

Palavras-chave: Diabetes Mellitus Tipo 1. Criança. Educação em Saúde. Enfermagem Pediátrica.

\section{Introdução}

O Diabetes Mellitus (DM) é uma das mais importantes doenças crônicas em esfera mundial que apresenta alta prevalência em todas as idades e au- menta a incidência de doenças cardiovasculares e complicações a longo prazo com limitação e risco de vida ao portador. ${ }^{1}$ A doença requer cuidados especiais e exige do paciente comportamento disciplinar, obediência na terapêutica e conhecimento de tudo que envol-
* O artigo originou-se de um trabalho de conclusão de Enfermagem do Centro Universitário Metodista do Sul (IPA), 2008.

1. Enfermeira. Graduada pelo Centro Universitário IPA Metodista do Rio Grande Sul. Local da pesquisa: Hospital Grupo Conceição - RS. E-mail: rubin_nilda@ hotmail.com

2. Docente, Centro universitário IPA Metodista do Rio Grande Sul - RS. E-mail: karina.azzolin@metodistadosul.edu.br

3. Enfermeira. Doutora do Centro de Pesquisa Clinica Hospital de Clinicas de Porto Alegre RS.
Correspondencia: Hospital de Clinicas de Porto Alegre (HCPA) Centro Cirúrgico Ambulatórial (CCA) Rua Ramiro Barcelos № 2350, Bairro Bom Fim

Porto Alegre /RS.

Artigo recebido em 10/03/2011 Aprovado para publicação em 1\%/12/2011 
va o tratamento. Este conjunto de fatores compõe a definição de adesão, onde vários componentes unidos formam medidas a serem aplicadas e cumpridas. ${ }^{2-4}$

Segundo a Organização Mundial de Saúde, a estimativa em 2000 era de 173 milhões de diabéticos, com projeção de 366 milhões para $2030 ;^{2}$ e a prevalência de pacientes com diabetes para todas as idades, e em todo mundo, será de 4,4\% em 2030. ${ }^{5}$

A DM possui duas categorias etiopatogenéticas: Diabetes Mellitus tipo 1 (DM 1), cuja causa é uma deficiência absoluta de secreção de insulina identificada por evidências sorológicas de um processo patológico auto-imune; e Diabetes Mellitus tipo 2 (DM 2), cuja causa é uma combinação de resistência à ação da insulina e resposta secretora de insulina compensatória inadequada, sendo suficiente para alterações funcionais em vários tecidos. ${ }^{6}$

Em crianças e adolescentes, a DM 1 tem crescido 3\% ao ano, elevando-se a 5\% entre crianças em fase pré-escolar. Estima-se que cerca de 70.000 crianças com menos de quinze anos desenvolva a doença. A estimativa prevista para 2030 é de que, em média, 200 crianças sejam diagnosticadas como portadoras de diabetes por dia. ${ }^{5}$ No Brasil, um estudo realizado em Londrina, Paraná, mostrou a incidência de 12,7/ $100.000^{7}$ e, no Rio Grande do sul, o laboratório do ICD estima que existam 8 mil crianças e adolescentes com DM 1 e a incidência de 312 novos casos diagnosticados anualmente. $^{8}$

Enfermidades crônico-degenerativas como a DM 1 requerem tratamento durante toda a vida e geram, na relação familiar, momentos difíceis para todos os membros, pois a atenção e o cuidado com a criança absorvem muita energia e tempo. Para a continuidade do tratamento, no entanto, são imprescindíveis os aspectos cognitivos do doente, as suas necessidades individuais e biopsicossociais e as atitudes de aceitação do paciente frente ao manejo do diabetes. ${ }^{4,9}$ Além disso, é necessário criar algum vínculo entre a família e os profissionais especializados, facilitando a interação com a equipe de saúde e motivando a prática do autocuidado. ${ }^{10}$

Quanto à não-adesão ao tratamento, a baixa escolaridade e a falta de conhecimento no manejo da doença são fatores importantes em virtude da complexidade terapêutica, o que também dificulta durante a abordagem dos profissionais. ${ }^{11}$

Este estudo tem por objetivo avaliar a adesão ao tratamento por crianças portadoras de Diabetes Mellitus tipo 1, em idade escolar, atendidas em um ambulatório especializado de Porto Alegre, identificar o conhecimento da criança e da família a respeito da doença e do tratamento, verificar as dificuldades encontradas pela criança durante todo o processo terapêutico e associar o conhecimento de crianças e pais sobre a doença diante do tempo de atendimento ambulatorial com relação ao tempo de diagnóstico.

\section{Método de pesquisa utilizado}

Este estudo transversal foi realizado no ambulatório do Instituto da Criança com Diabetes, situado no Grupo Hospitalar Conceição de Porto Alegre, Rio Grande do Sul, onde é desenvolvido o Programa de Assistência às crianças portadoras de diabetes.

A amostra foi composta por 25 crianças portadoras de Diabetes Mellitus tipo I com idade escolar entre 6 e 12 anos atendidas no serviço referido por, ao menos, seis meses. O período de estudo da amostra compreendeu dois meses ano de 2008.

A avaliação do conhecimento da doença e das dificuldades no tratamento foi realizada através da aplicação de um questionário (Apêndice A) à criança e ao seu responsável, utilizando questões como: "O que é DM1?", "Quais as complicações?", "Como identificar sinais e sintomas de hipoglicemia e hiperglicemia?", "Quais as dificuldades encontradas durante o tratamento?", entre outras. Para auxiliar a criança a identificar o que faz parte de seu dia-a-dia, tanto na alimentação, quanto nas atividades físicas, foram utilizadas figuras ou fotos de alimentos e de atividades como natação, futebol, vídeo-game, TV, computador e caminhadas. ${ }^{12}$

A observação e o acompanhamento de 10 consultas de enfermagem também fizeram parte da coleta de dados. O objetivo desse acompanhamento foi de verificar as questões discutidas durante as consultas com o enfermeiro, principalmente quando o assunto estivesse diretamente relacionado ao tratamento da DM1.

As variáveis estudadas foram: o perfil sóciodemográfico e perfil clínico, o conhecimento sobre a doença, as dificuldades encontradas durante o tratamento, o plano alimentar e a prática de exercícios físi$\cos$.

Foram consideradas aderentes ao tratamento as crianças que: não faltaram a nenhuma consulta nos últimos seis meses, não esqueceram de aplicar insulina nenhuma vez no último mês e seguiram a dieta prescrita. 
O questionário foi aplicado à criança - acompanhada de seu familiar, em um local arejado e calmo. O tempo médio da aplicação do questionário foi de 15 minutos.

\section{Considerações éticas}

O estudo foi aprovado pelo comitê de ética do Instituto da Criança com Diabetes. O Termo de Consentimento Livre Esclarecido (TCLE) foi assinado pelos pais ou responsável e, inclusive, pela criança. Foram observados os cuidados éticos conforme a Resolução 196/96 do Conselho Nacional de Saúde sobre Diretrizes e Normas Regulamentadoras de Pesquisa Envolvendo Seres Humanos, respeitando principalmente o item IV, sobre pesquisas que envolvam crianças e adolescentes.

\section{Análise estatística}

A análise dos dados foi realizada com auxílio do programa SPSS para Windows versão 12.0 utilizando estatística descritiva, porcentagens, distribuição de freqüências, média e desvio padrão.

\section{Resultados}

Os dados sócio-demográficos estão descritos na Tabela 1.

A pesquisa procurou saber o tempo de vínculo com o ambulatório e encontrou um tempo de atendimento ambulatorial de $1,76 \pm 0,7$ e tempo em doença $1,96 \pm 0,8 \mathrm{O}$ tempo foi quase o mesmo, sendo importante que o processo de preparo para a terapêutica seja no início do diagnóstico para que futuros agravos sejam evitados. ${ }^{13}$

Quanto à dependência dos pais, $84 \%$ das crianças ainda não tinham responsabilidade pelo cuidado de si. No aspecto de conhecimento sobre a doença pela criança, $44 \%$ dos participantes reconheceram e souberam explicar sobre a sua patologia, respondendo, de forma simplificada, que diabetes é a diminuição de insulina ou a não produção de insulina pelo pâncreas e que é necessário repor a insulina no organismo.

Trinta e seis por cento das crianças necessitaram utilizar de artefatos comparativos a objetos animados para tentar explicar o seu conhecimento a respeito da doença. Apenas $20 \%$ não possuíam conhecimento suficiente ou não conseguiram se expressar de alguma forma. Quanto à continuidade do tratamento, $72 \%$ dos pais referiram a dificuldade de acesso ao centro ICD e ao fato de residirem fora de Porto Alegre. $68 \%$ dos responsáveis também apontaram como problema o alto custo do tratamento.

Quanto à ingesta alimentar, 48\% dos casos relataram haver uma limitação em seguir o plano alimentar indicado. Alguns pais referiram ser necessário mudar alimentação de toda a família em virtude da criança, mas que isso nem sempre era possível, por razões econômicas. Da mesma forma, os pais também relataram perder o controle da ingesta alimentar das crianças no período em que elas estão dentro da escola.

Onze das vinte e cinco crianças (44\%) foram consideradas aderentes ao tratamento no período de seis meses em que estavam sendo acompanhadas no ICD, conforme demonstra a Tabela 2 .

Quando comparadas as idades dos aderentes e não-aderentes, a média foi de 8 e 9 anos, respectivamente, não havendo diferença estatisticamente significativa. Dez pacientes (40\%) seguem o plano alimentar orientado pela nutricionista e supervisionado pela enfermagem. Todas as 25 crianças analisadas referiram ingerir três alimentos em comum: feijão, arroz e pão francês. Quanto ao tipo de carne, $12 \%$ optaram por carne de gado, $4 \%$ por frango, $80 \%$ marcaram as

Tabela 1

Perfil sócio-demográfico de 25 crianças portadoras de DM1 atendidas no ambulatório

\begin{tabular}{lcc}
\hline Características & Média \pm DP & $\%$ \\
\hline Idade (anos) & $09 \pm 1,7$ & 60 \\
Sexo (masculino) & 09 & 72 \\
Interior do Estado & 18 & \\
Tempo de atendimento no ambulatório (em anos) & $1,76 \pm 0,7$ & \\
Tempo de Doença DM 1 (em anos) & $1,96 \pm 0,8$ & \\
\hline
\end{tabular}


Tabela 2

Adesão ao tratamento, referente a consultas e aplicação de insulina

\begin{tabular}{lcc}
\hline Frequência & Número & $(\%)$ \\
\hline Não faltou nenhuma consulta & 19 & $(76)$ \\
Faltou uma consulta & 05 & $(20)$ \\
Faltou duas ou mais consultas & 01 & $(04)$ \\
Esqueceu de aplicar uma vez a insulina & 02 & $(08)$ \\
\hline
\end{tabular}

duas alternativas e $4 \%$ referiu não comer carne. As saladas, verduras e legumes foram opção de $72 \%$, e salada de frutas de $64 \%$. Outros alimentos não indicados para pacientes portadores de DM foram referidos por grande parte das crianças como: pastéis $60 \%$, pizza $48 \%$, batatas-fritas $80 \%$, cachorro-quente $36 \%$, bombons $28 \%$, hambúrguer $12 \%$ e biscoito recheado $16 \%$.

Os refrigerantes light/diet foram selecionados como opção de preferência das 25 crianças, seguida pelas seguintes opções ordenadas de acordo com a preferência das crianças: sucos naturais, água sem gás, refrigerante normal e água com gás.

Quanto à atividade física, foi evidenciado que 96\% das crianças realizavam algum tipo de atividade aeróbica, sejam exercícios físicos ou brincadeiras. Cinquenta e dois por cento desses pacientes realizavam mais de duas vezes por semana algum tipo de exercício físico. As atividades preferidas foram: corrida $96 \%$ e, na segunda opção, jogar futebol $72 \%$. As atividades desenvolvidas regularmente pelas crianças estão apresentadas na curva, conforme suas preferências, no Gráfico 1.

\section{ATIVIDADES REALIZADAS SEGUNDO A PREFERÊNCIA}

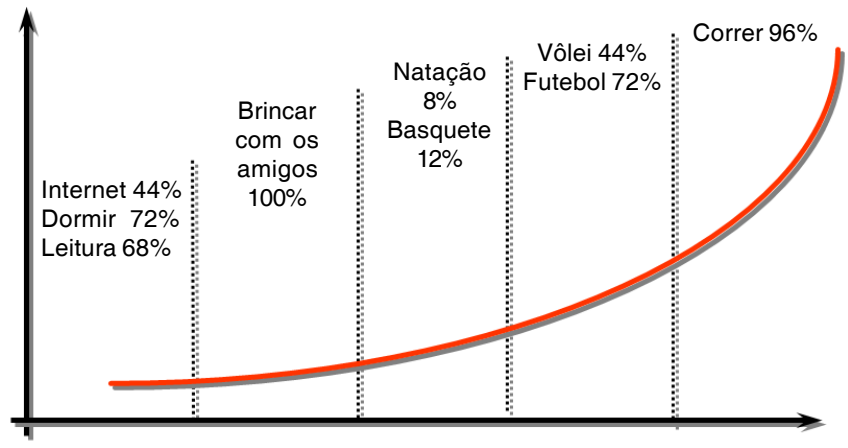

ATIVIDADES AERÓBICAS

Gráfico 1: Atividades realizadas pelas crianças segundo suas preferências
Quanto às observações realizadas nas dez consultas de enfermagem, as questões sobre insulina e peso da criança foram abordadas em 100\% destas pelas enfermeiras, assim como a realização do exame físico, conforme demonstrado no Gráfico 2.

Sobre a importância das consultas de Enfermagem, 92\% das crianças e seus responsáveis responderam que é importante em virtude das atividades educativas realizadas durante a consulta, como a técnica de aplicação da insulina, o rodízio de locais de aplicação e exame físico.

\section{Discussão}

Pesquisas sobre aderência aos tratamentos de doenças crônicas permitem identificar vários fatores responsáveis à continuidade do tratamento. A publicação do artigo Adherence to Long-Term Therapies Evidences for actives ${ }^{3}$ pela Organização Mundial de Saúde (OMS) discute a adesão ao tratamento do diabetes e aponta mais de um fator para avaliar esse processo discutindo que a terapêutica farmacológica em si não é capaz de garantir a estabilidade da doença. A isso agrega um conjunto de informações a ser trabalhado com os pacientes em virtude da complexidade da doença e orienta que se deve procurar manter o paciente mais próximo de seu padrão normal de vida para que consiga seguir o tratamento. Todos os processos como: o controle da glicemia, dieta alimentar, práticas de atividades físicas, uso da medicação de forma correta, o autocuidado, família, processo sócioeconômico e outros, funcionam de forma independente e são indispensáveis para o tratamento. $\mathrm{O}$ resultado deste conjunto é a qualidade de vida do paciente.

A média de idade dos pacientes em nosso estudo que já autoaplicavam sua medicação foi de nove anos de idade, o que vai ao encontro dos dados encontrados na literatura, que apontam que a transferência de responsabilidade dos pais em relação ao fi- 


\section{№ DE CONSULTAS}

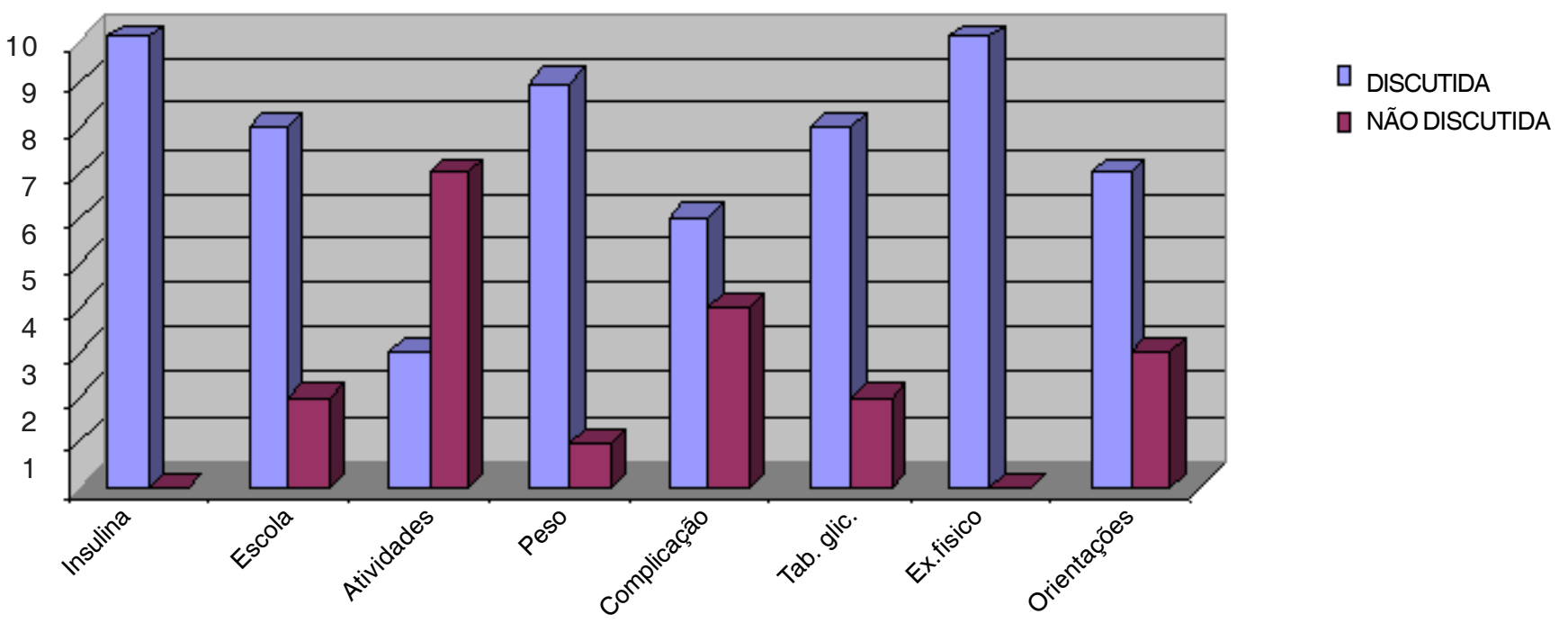

ASSUNTOS ABORDADOS

Gráfico 2: Assuntos abordados da consulta de enfermagem.

lho - no que concerne à auto-aplicação de insulina ocorre por volta dos dez aos doze anos de idade. ${ }^{14}$

A maioria das crianças teve a capacidade de identificar sinais e sintomas de complicações como hipohiperglicemia. A pesquisa mostrou que, quando questionadas sobre o conhecimento com relação à DM 1, apenas $12 \%$ das crianças não souberam responder e $8 \%$ não lembravam quais eram as complicações. Embora as práticas educativas em saúde tenham estado presentes no cotidiano dos profissionais da enfermagem, ainda existem algumas dificuldades que a enfermagem não está preparada para lidar, o que limita o processo de aprendizado da criança (considerando a dificuldade de compreensão que o adulto possa ter com relação à doença e a dificuldade que a criança possa vir a ter), sendo necessárias diferentes técnicas de ensino para garantir a aprendizagem, como a utilização de diferentes ferramentas nos métodos educacionais. ${ }^{4}$

O objetivo para a obtenção da adesão ao tratamento da criança com DM 1 é melhorar o controle metabólico através de uma terapêutica alimentar, monitoramento da glicemia capilar e exercícios físicos

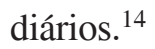

Na perspectiva de melhorar a qualidade de vida, as atividades diárias de exercícios físicos trazem grandes benefícios no que se refere à prevenção de doenças cardiovasculares para o futuro dessas crianças. ${ }^{15}$ Quanto à frequência dos exercícios físicos em nosso estudo, foi possível constatar que $12 \%$ das crianças realizavam de uma a duas vezes por semana alguma atividade física, $56 \%$ faziam atividades mais de duas vezes por semana e $32 \%$ praticavam apenas na escola. Outro estudo em que foram avaliadas 66 crianças com idade suficiente para realizar atividades físicas, mostrou que 53,5\% exercitavam-se regularmente, e a atividade física mais freqüente foi a prática de futebol 39,4\%.16. Da mesma forma, os nossos resultados também apontaram o futebol como preferência, porém, a atividade mais realizada foi a corrida (96\%). Segundo a Sociedade Brasileira de Pediatria, as atividades físicas podem iniciar em qualquer idade, desde que essas atividades estejam adequadas ao paciente. Quando iniciadas na fase pré-escolar, estimulam o desenvolvimento da força muscular, flexibilidade e resistência, aperfeiçoamento a coordenação motora, estimula metabolismo ósseo, aumenta capacidade respiratória e cardíaca, melhora o humor e o apetite, previne obesidade, diminui o risco de hipertensão, diabetes e cardiopatia isquêmica. ${ }^{16}$

Em nosso estudo os resultados mostraram que $60 \%$ das crianças apresentavam dificuldades em seguir o plano alimentar. As crianças não restringiam alguns alimentos da dieta comendo pastéis, pizza, batatas-fritas, cachorro-quente, bombons, hambúrguer e biscoito-recheado. A mesma dificuldade foi evidenciada em um estudo com adultos realizado nos Estados 
Unidos. Neste estudo, de $70 \%$ a $75 \%$ dos participantes relataram que não seguem um plano alimentar ${ }^{3}$; porém, o resultado de um estudo na Finlândia sobre a adesão às recomendações dietéticas foi elevado: $70 \%$ dos participantes adultos informaram ter uma refeição principal regular, conforme orientações prescritas. ${ }^{2,17}$

A Sociedade Brasileira de Diabetes (SBD) e o Ministério da saúde elaboraram um manual de contagem de carboidratos como medida de inclusão social e como ferramenta para facilitar a escolha dos alimentos durante o controle da DM. Em suas recomendações aconselha a utilização de três porções de frutas e duas porções de legumes e verduras todos os dias como dieta adequada para DM.

Ficou evidenciado que as crianças sentiam-se mais seguras quanto ao tratamento quando avaliadas pelas enfermeiras, onde vários aspectos foram abordados individualmente como: rodízio dos locais de aplicações de insulina, orientações no controle da glicemia capilar e orientação da dieta alimentar, proporcionando assim, maior integração entre o profissional, pacientes e familiares. Este fato reforça a idéia de que através da educação aos portadores de diabetes é possível conseguir reduções importantes das complicações e, conseqüentemente, melhoria da qualidade de vida, o que se traduz como adesão ao tratamento. ${ }^{4}$

A porcentagem encontrada em nosso estudo quanto à adesão ao tratamento foi de $44 \%$, o que vai ao encontro dos resultados divulgado pela OMS, onde se descreve que a aderência ao tratamento das doenças crônicas ainda é pobre, sendo um problema mundial. ${ }^{18} \mathrm{~A}$ aderência à terapia em longo prazo para doenças crônicas em países subdesenvolvidos representa taxas ainda mais baixas. ${ }^{3}$

\section{Conclusão}

O objetivo do estudo foi verificar a adesão ao tratamento em crianças portadoras de DM $1 \mathrm{em}$ um ambulatório no ICD. Os resultados encontrados em nosso estudo foram comparáveis aos índices de adesão ao tratamento do DM divulgados pela Organização Mundial da Saúde (OMS). As dificuldades que influenciaram o índice de adesão foram o deslocamento até a instituição, o alto custo do tratamento e as dificuldades de manter a dieta, pois inclui mudanças e reestruturação do cardápio alimentar de toda a família. Quanto ao trabalho realizado pelos enfermeiros nas consultas de enfermagem houve pleno reconhecimento dos pais e crianças.

O diabetes Mellitus infantil é considerado um problema de saúde pública, sendo responsável pela morbi-mortalidade de grande parte da população. Atualmente, a literatura tem apontado suas complicações como um grave problema e com sérios riscos à vida. Também é válido dizer que estas complicações podem ser evitadas através do monitoramento e a partir da educação em saúde.

O envolvimento da família com os profissionais de saúde no processo de cuidado produz um papel fundamental na adesão ao tratamento das crianças em idade escolar. Esse processo de aprendizado que a Enfermagem realiza através das consultas resulta na conscientização, na mudança de comportamento e, muitas vezes, no estilo de vida dessas famílias. As medidas propõem o controle da doença e prevenção de futuras complicações, realizando ações positivas que possibilitem conquistar a autoestima, a vontade de aprender e conviver com a diabetes.

\section{ABSTRACT}

Diabetes Mellitus is considered one of the most important endocrine-metabolic diseases in childhood, with limitation and risk of life to the carrier in reason of its cardiovascular complications at long term. This is a quantitative study which aimed to evaluate the adherence to the treatment of children in school age who are carriers of Diabetes Mellitus Type 1 and are treated at specialized clinic in Porto Alegre: the Institute for Children with Diabetes (ICD). Data were collected through a semi-structured questionnaire with 25 children with a mean age of 9 years old. The follow-up in the clinic was $1.96 \pm 0.8$ years, and the mean of the treated disease was $1.76 \pm 0.7$ years. Most children 21 (84\%) have parents as the primary caregiver, all 25 $(100 \%)$ recognize the importance of the nursing treatment and $23(92 \%)$ trust in the conduct of professionals. Fifteen $(60 \%)$ children face difficulties related to the food plan. The adherence to the treatment was observed in 11 (44\%) children, who did not miss any appointment; and they do not forget to apply insulin in the last 6 months and reported that they follow the prescribed diet. Conclusion: The adherence to the treatment of diabetics children is related to the parents interest and knowledgement of disease in addition to socio-economic factors. The nurse's work was fully recognized by the children's parents.

Keywords: Diabetes Mellitus, Type 1. Child. Health Education. Pediatric Nursing. 


\section{Referências bibliográficas}

1. Galindo V, Cavalcanti N, Lyra R. Definição, diagnóstico e classificação dos distúrbios no metabolismo dos hidratos de carbono. In: Lyra R, Cavalcanti N, (Org.). Diabetes mellitus. Rio de Janeiro: Diagraphic, 2006.

2. Moreira LB, Fernando PF, Monte FS, Galvão RI, Martins, AM. Conhecimento sobre tratamento farmacológico em pacientes com doenças renal crônica. Rev Bras Cienc Farm. 2008; 44: 315-25.

3. Burkhart PV, Sabaté E. Adherence to long-term therapies: evidence for action. J Nurs Scholarsh. 2003; 35: 207.

4. Cazarini RP, Zanetti ML, Ribeiro KP, Pace AE, Foss MC. Adesão a um grupo educativo de pessoas portadoras de diabetes mellitus: porcentagem e causas. Medicina (Ribeirão Preto). 2002; 35: 142-50.

5. World Health Organization. Diabetes Day-year of the child. World Diabetes Day. [Online], [acesso em 2007, Nov. 17] Disponível em [http//www.worlddiabetesday.org/go/wdd.htm]

6. Expert Committee on the Diagnosis and Classification of Diabetes Mellitus. Report of the expert committee on the diagnosis and classification of diabetes mellitus. Diabetes Care. 2003; 26: 3160-7.

7. Rodrigues TM, Silva IN. Estatura final de pacientes com Diabetes Mellitus do tipo 1. Arq Bras Endocrinol Metab. 2001; 45: 108-14.

8. Tschiedel B, Ce GV, Geremia C, Mondadori P, Speggiorin S, Puñales MK. Organização de um serviço de assistência ao paciente com Diabetes Melito Tipo 1. Arq Bras Endocrinol Metab. 2008; 52: 219-32.

9. Nunes MD, Dupas G, Ferreira NM. Diabetes na infância/adolescência: conhecendo a dinâmica familiar. Rev Eletrônica Enferm. 2007; 7: 179-84.
10. Ferraz AE, Zanetti ML, Brandão EC, Campanelli LR, Foss MC, Paccola G, et al. Atendimento multiprofissional ao paciente com diabetes mellitus no Ambulatório de Diabetes do HCFMRPUSP. Medicina (Ribeirão Preto). 2000; 33: 170-5.

11. Sociedade Brasileira de Diabetes e Metabologia. Atualização brasileira sobre diabetes / Sociedade Brasileira de Diabetes. Rio de Janeiro: Diagraphic, 2005. 140p.

12. Minayo MC. O desafio do conhecimento: pesquisa qualitativa em saúde. 10 ed. São Paulo: Hucitec; 2007.

13. Sociedade Brasileira de Diabetes. Tratamento de crianças e adolescente com diabetes mellitus tipo1. In: Gomes MB, Dib AS (Org.). Diretrizes da sociedade Brasileira de Diabetes. Tratamento e acompanhamento Diabetes Mellitus. Sociedade Brasileira de Diabetes, São Paulo: 2006; 60-5.

14. da Costa PC, Franco LJ. Introdução da sacarose no plano alimentar de portadores de diabetes mellitus tipo 1: sua influência no controle glicêmico. Arq Bras Endocrinol Metab. 2005; 49(3): 403-9.

15. Vieira MA, Lima RA. Crianças e Adolescentes com doenças crônicas: vivendo com mudanças. Rev Latinoam Enferm. 2002; 10: 552-60.

16. Murahovschi J. Atividade Física na Infância (revisado em fevereiro/2011). Sociedade Brasileira de Pediatria. Diposnível em: [http://www.sbp.com.br/show_item2.cfm?id_categoria= 24\&id_detalhe=1242\&tipo_detalhe=s] [acesso em $2011 \mathrm{Nov}$ 10]

17. Sociedades Brasileira Diabetes. Manual oficial de contagem de carboidratos regional / Sociedade Brasileira de Diabetes, Departamento de Nutrição. Rio de Janeiro: Dois C: Sociedade Brasileira de Diabetes, 2009.

18. Silveira VM, Menezes AM, Post CL, Machado EC. Uma amostra de pacientes com diabetes tipo 1 no sul do Brasil. Arq Bras Endocrinol Metab. 2001; 45: 433-40. 


\section{ANEXO 1}

\section{Questionário ao Paciente}

\section{Perfil da criança}

Idade:

Sexo:

Residência:

Número irmãos:

Escolaridade:

Comorbidades e história familiar:

Tempo DM 1: ( ) 1-5anos ( ) $5-7$ anos ( ) $7-11$ anos

Tempo atendimento no ambulatório: ( ) menos 1 ano ( ) 1- 2 anos ( ) mais de 2 anos

Cuidador:

Tipo tratamento:

Quem aplica insulina:

\section{Adesão e Conhecimento sobre a doença}

1. O que é DM 1 ?

2. Quais as complicações:

3. Escolha das figuras abaixo os sinais e sintomas hipoglicemia e hiperglicemia:

4. O tratamento é importante?

5. A consulta com a enfermeira é importante por quê?

6. Dificuldades:

Sim ( ) Quais:

Não ( )

7. Faltou alguma consulta nos últimos 6 meses? Por quê?

8. Esqueceu de usar insulina alguma vez no último mês? Por quê?

9. Teve hipoglicemia ou hiperglicemia no último mês? Por quê?

10. Segue uma dieta especial: sim ( ) não( ).

Se possuir uma dieta especial o que não é permitido comer?

11. Das figuras (mostradas a seguir) selecione os alimentos que você consome - no almoço:

- no lanche da escola: 
1. ( )

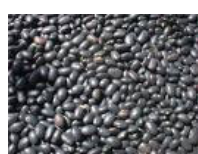

2. ( )

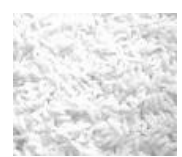

3. ( )

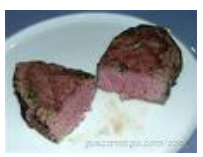

4. ( )

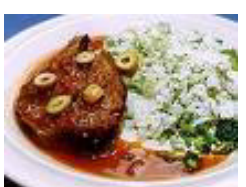

7. ( )

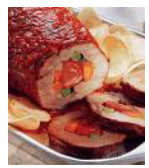

8. ( )

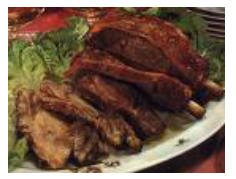

9 ( )

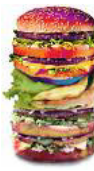

10. ( )

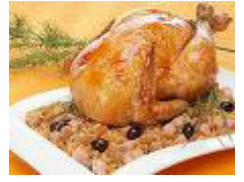

11. ( )

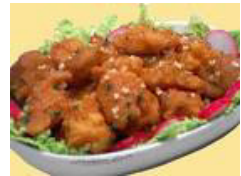

12. ( )

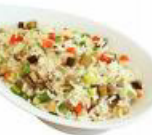

13. ( )

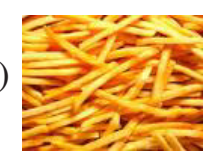

$14($ )

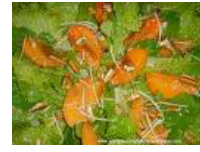

1. ( )

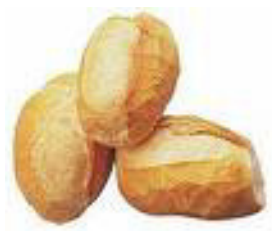

5. ( )

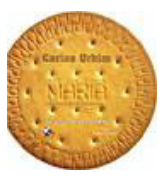

5. ( )

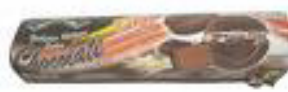

2. ( )

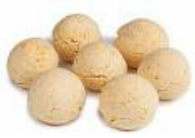

16. ( )

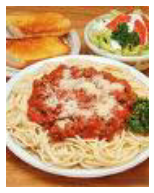

3. ( )

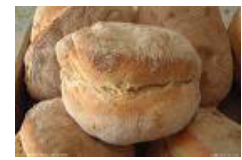

7. ( )

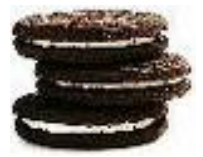

17. ( )

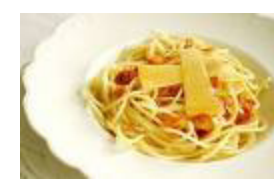

4. ( )

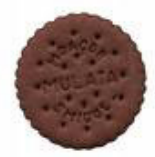

8. ( )

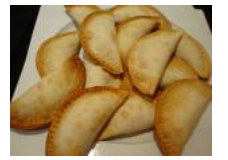

11. ( )

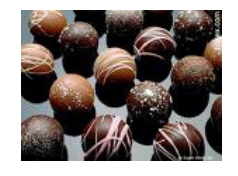

12. ( )
13. ( )

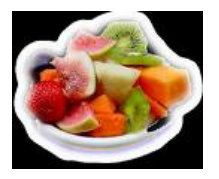

14. (

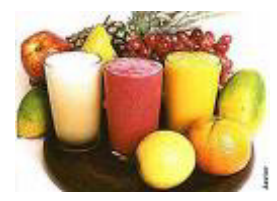

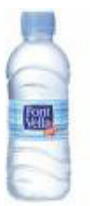

15. ( )

a) ( ) sempre Light, zero caloria, diet.

16. ( )

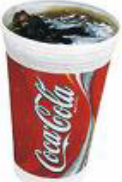

17. ( )

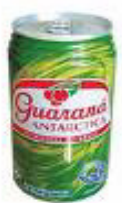

b) ( ) normal 
16. ( ) não faz lanche

17. ( ) não come nada na escola

18. ( ) eventualmente faz lanche na escola

III. Das figuras abaixo selecione as atividades que você gosta de fazer:

1. ( )

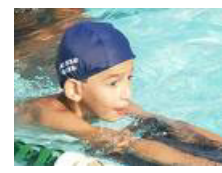

natação
2. ( )

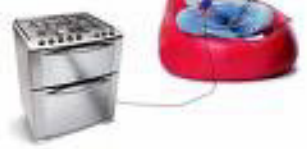

jogos virtuais
3. ( )

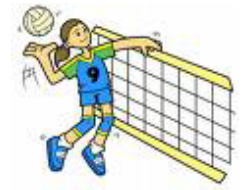

vôlei

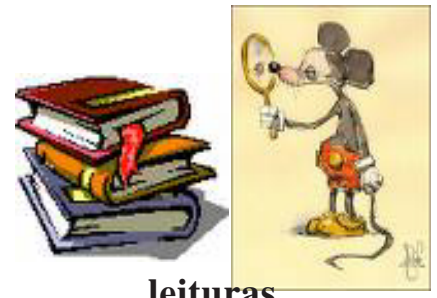

leituras
4. ( )

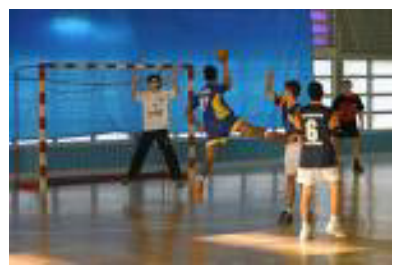

handebol

8. ( )

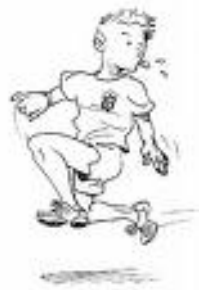

correr futebol

9. ( )

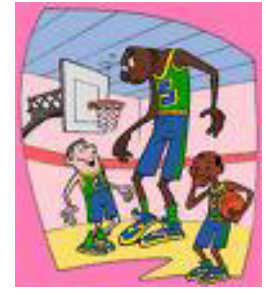

basquete
10. ( )

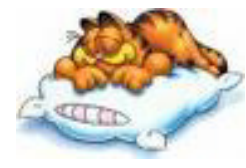

dormir
11. ( )

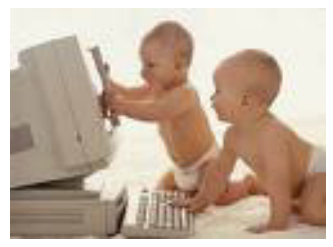

Internet

\section{Observação consulta de enfermagem}

Consulta:

Discutiu:

- Sobre a doença

- Sobre a dieta

- Sobre o controle da glicemia

- Aplicação insulina

- Sobre a escola

- Sobre as atividades diárias

- Sobre o peso

- Sobre as complicações

- Trouxe tabela com valores de glicemia

- Realizou exame físico

- Orientações

- Encaminhamento: Sim: Não:

- Para Quem

- Outros

Não discutiu:

\section{2. ( prefere não}

